\title{
Dexamethasone normalizes aberrant elastic fiber production and collagen 1 secretion by Loeys-Dietz syndrome fibroblasts: a possible treatment?
}

\author{
Christopher P Barnett ${ }^{1}$, David Chitayat ${ }^{1,2}$, Timothy J Bradley ${ }^{3}$, Yanting Wang ${ }^{4}$ and Aleksander Hinek ${ }^{\star, 4}$ \\ Loeys-Dietz syndrome (LDS) is an autosomal dominant connective tissue disorder characterized by facial dysmorphism, \\ cleft palate, dilation of the aortic arch, blood vessel tortuosity and a high risk of aortic dissection. It is caused by mutations \\ in the transforming growth factor $\beta$-receptor 1 and 2 (TGF $\beta$-R1 and TGF $\beta$-R2) genes. Fibroblasts derived from 12 Loeys-Dietz \\ syndrome patients, six with TGFB-R1 mutations and six with TGFB-R2 mutations, were analyzed using RT-PCR, biochemical \\ assays, immunohistochemistry and electron microscopy for production of elastin, fibrillin 1, fibulin 1 and fibulin 4 and \\ deposition of collagen type I. All LDS fibroblasts with TGF $\beta$-R1 mutations demonstrated decreased expression of elastin \\ and fibulin 1 genes and impaired deposition of elastic fibers. In contrast, fibroblasts with TGF $\beta$-R2 mutations consistently \\ demonstrated intracellular accumulation of collagen type $I$ in the presence of otherwise normal elastic fiber production. \\ Treatment of the cell cultures with dexamethasone induced remarkable upregulation in the expression of tropoelastin, \\ fibulin 1- and fibulin 4-encoding mRNAs, leading to normalization of elastic fiber production in fibroblasts with TGF $\beta$-R1 \\ mutations. Treatment with dexamethasone also corrected the abnormal secretion of collagen type I from fibroblasts with \\ TGF $\beta$-R2 gene mutations. As the organogenesis-relevant elastic fiber production occurs exclusively in late fetal and early \\ neonatal life, these findings may have implications for treatment in early life. Further studies are required to determine \\ if dexamethasone treatment of fetuses prenatally diagnosed with LDS would prevent or alleviate the connective tissue and \\ vascular defects seen in this syndrome.
}

European Journal of Human Genetics (2011) 19, 624-633; doi:10.1038/ejhg.2010.259; published online 26 January 2011

Keywords: Loeys-Dietz syndrome; fibroblasts; elastic fibers; collagen; dexamethasone

\section{INTRODUCTION}

Loeys-Dietz syndrome (LDS types 1A, 1B, 2A and 2B, OMIM \#609192, \#610380, \#610168 and \#608967, respectively) is a recently described autosomal dominant connective tissue disorder caused by mutations in the transforming growth factor $\beta$-receptor 1 and 2 genes (TGF $\beta$-R1 and TGF $\beta$-R2). ${ }^{1}$ The TGF $\beta$-R1 and R2 receptors belong to the transmembrane-spanning protein serine/threonine kinase family and control several cellular processes including growth inhibition, apoptosis, proliferation and extracellular matrix production. ${ }^{2,3}$ LDS has phenotypic overlap with Marfan syndrome (MFS, OMIM \#154700), caused by mutations in the FBN1 gene (OMIM \#134797) but LDS patients have a higher risk of aortic dissection. ${ }^{1}$ Life expectancy of LDS patients is significantly shortened when compared with MFS.

The most common features of LDS type 1 are hypertelorism, cleft palate with bifid uvula, aortic root aneurysm, arterial tortuosity, arachnodactyly, pectus deformity and joint laxity, with some patients having craniosynostosis, retrognathia and blue sclerae. Patients classified as LDS type 2 do not have cleft palate, craniosynostosis or hypertelorism but demonstrate features in common with EhlersDanlos syndrome type IV (EDS IV, OMIM\#130050, vascular type) such as increased joint laxity, easy bruising, translucent skin, distended scars and spontaneous rupture of the spleen and bowel. ${ }^{4-7}$ However, because of the relatively small number of fully analyzed cases bearing the same individual mutations, no consensus has been reached whether the presence of particular mutations could be exclusively linked to the given feature observed in both subtypes of LDS.

The existence of significant phenotypic overlap between LDS and MFS suggests that the causative mechanism may be similar in both conditions, despite the fact that they are caused by mutations in different genes. It has been suggested that aberrant TGF $\beta$ signaling might contribute to the connective tissue pathology in LDS as it does in MFS, where the inherited deficiency of fibrillin-1 protein contributes to intensified TGF $\beta$-induced signals. ${ }^{8-10}$ The molecular mechanism leading to aortic dissections in LDS has not been fully characterized, but elastic fiber fragmentation and abnormal collagen deposition was demonstrated in aortic explants from LDS patients. ${ }^{11}$

In our study, gene expression analysis, biochemical assays, immunohistochemistry and electron microscopy (EM) were used to compare the production of four major elastic fiber components (elastin, fibrillin 1, fibulin 1 and fibulin 4) and the production of collagen type I in fibroblasts derived from 12 LDS patients with different mutations in both TGF $\beta$-R1 and TGF $\beta$-R2. We also explored

${ }^{1}$ Division of Clinical and Metabolic Genetics, The Hospital for Sick Children, University of Toronto, Toronto, ON, Canada; ${ }^{2}$ The Prenatal Diagnosis and Medical Genetics Program, Department of Obstetrics and Gynecology, Mount Sinai Hospital, University of Toronto, Toronto, ON, Canada; ${ }^{3}$ Division of Cardiology, The Hospital for Sick Children, University of Toronto, Toronto, ON, Canada; ${ }^{4}$ Division of Cardiovascular Research, The Hospital for Sick Children, University of Toronto, Toronto, ON, Canada

*Correspondence: Dr A Hinek, Division of Cardiovascular Research, The Hospital for Sick Children, 555 University Avenue, Toronto, ON, Canada M5G 1X8. Tel: +1 416 813 6725; Fax: +1 416813 7480; E-mail: alek.hinek@sickkids.ca

Received 9 August 2010; revised 9 December 2010; accepted 22 December 2010; published online 26 January 2011 
whether dexamethasone, which directly interacts with the elastin gene promoter to induce transcription of tropoelastin mRNA, ${ }^{12}$ would improve production of elastic fibers in the cultures of LDS dermal fibroblasts.

\section{MATERIALS AND METHODS}

\section{Cell cultures}

Primary cultures of dermal fibroblast derived from six LDS patients bearing different mutations of TGF $\beta$-R1 (S241L, R487Q, C722T, T200I, M318R and G1460A) and from six patients with different TGF $\beta$-R2 mutations (A355P, R528H, R495X, P427L, R460L and A329T) were used in this study. The fibroblasts were collected from skin biopsies taken for diagnostic purposes or surgery at the Hospital for Sick Children in Toronto, Canada and at the Johns Hopkins Medical Center, USA (donated by Dr H Dietz). The respective institutional review board approvals and patient informed consents were obtained. The guidelines for the protection of human subjects of the Department of Health and Human Services and of the Declaration of Helsinki were strictly followed. All fibroblasts (passages 1-6) were initially plated (100000 cells/dish and maintained in Dulbecco's modified eagle's medium (DMEM) supplemented with $1 \%$ antibiotics/antimycotics, and $2 \%$ of FBS in the presence and absence of $10 \mu \mathrm{m}$ dexamethasone (Sigma, St Louis, MO, USA). The selected dose produced an optimal elastogenic effect in cultures of normal human skin fibroblasts. It also corresponds well to transient concentration detected in the serum of patients treated with therapeutic doses of this glucocorticoid $(5-15 \mathrm{mg} / \mathrm{kg})$. The cultures were then used for the quantitative assessment of mRNAs encoding major components of elastic fibers and collagen type 1, quantification of new insoluble elastin production, for the EM and for light microscopy immunohistochemical evaluation of elastin, fibrillin 1, fibulin 1, fibulin 4 and collagen type I deposition.

\section{One-step reverse transcriptase-polymerase chain reaction (RT-PCR) analysis}

Confluent fibroblast cultures were treated with or without $10 \mu \mathrm{M}$ dexamethasone for $24 \mathrm{~h}$. Total RNA was extracted using the RNeasy Mini Kit (Qiagen, Valencia, CA, USA) according to the manufacturer's instruction. In each experiment, $0.5 \mu \mathrm{g}$ of total RNA was added to each one-step RT-PCR (Qiagen One-Step RT-PCR Kit), and reactions were set up according to the manufacturer's instructions in a total volume of $25 \mu \mathrm{l}$. The reverse transcription step was performed for human elastin, fibrillin 1 , fibulin 1, fibulin 4, collagen 1 and glyceraldehyde-3-phosphate dehydrogenase (GAPDH) reactions at $50{ }^{\circ} \mathrm{C}$ for $30 \mathrm{~min}$, followed by $15 \mathrm{~min}$ at $94^{\circ} \mathrm{C}$. The elastin PCR reaction was performed under the following conditions: 28 cycles at $94{ }^{\circ} \mathrm{C}$ denaturation for $45 \mathrm{~s}, 63^{\circ} \mathrm{C}$ annealing for $45 \mathrm{~s}, 72^{\circ} \mathrm{C}$ extension for $1 \mathrm{~min}$ and 1 cycle at $72{ }^{\circ} \mathrm{C}$ final extension for $10 \mathrm{~min}$. The fibrillin 1 PCR reaction included: 26 cycles at $94{ }^{\circ} \mathrm{C}$ denaturation for $30 \mathrm{~s}, 52^{\circ} \mathrm{C}$ annealing for $30 \mathrm{~s}, 72{ }^{\circ} \mathrm{C}$ extension for $1 \mathrm{~min}$ and 1 cycle at $72{ }^{\circ} \mathrm{C}$ final extension for $10 \mathrm{~min}$. The fibulin- 1 and fibulin- 4 PCR reactions included: 28 cycles at $94^{\circ} \mathrm{C}$ denaturation for $30 \mathrm{~s}, 60^{\circ} \mathrm{C}$ annealing for $30 \mathrm{~s}, 72{ }^{\circ} \mathrm{C}$ extension for $1 \mathrm{~min}$ and $1 \mathrm{cycle}$ at $72{ }^{\circ} \mathrm{C}$ final extension for $10 \mathrm{~min}$. The pro- $\alpha 1$ (collagen I) chain PCR included 20 cycles of $94^{\circ} \mathrm{C}$ denaturation for $30 \mathrm{~s}, 58^{\circ} \mathrm{C}$ annealing for $30 \mathrm{~s}, 72^{\circ} \mathrm{C}$ extension for $10 \mathrm{~min}$ and $1 \mathrm{cycle}$ of $72^{\circ} \mathrm{C}$ final extension for $10 \mathrm{~min}$. GAPDH PCR reaction included 21 cycles of $94^{\circ} \mathrm{C}$ denaturation for $20 \mathrm{~s}, 58^{\circ} \mathrm{C}$ annealing for $30 \mathrm{~s}, 72^{\circ} \mathrm{C}$ extension for $1 \mathrm{~min}$ and 1 cycle of $72^{\circ} \mathrm{C}$ final extension for $10 \mathrm{~min}$. The primers used are listed in Table 1 . The PCR products from each reaction were run on a $1 \%$ agarose gel and stained with ethidium bromide. The dose curves were also created to validate the quantitative assay of each tested product. In each presented case, three separate reactions were performed and the amounts of mRNAs encoding particular ECM component-related product were normalized to the amount of GAPDH mRNA.

\section{Real-time RT-PCR}

For comparison, the RNA samples extracted with Rneasy Mini Kit (Qiagen) from cells of normal individuals and LDS patients were subjected to the reverse transcription proceeded with $1 \mu \mathrm{g}$ of total RNA using SuperScript II First-Strand Synthesis System (Invitrogen). The quantitative real-time PCR reactions were then performed using SYBR Green Master Mix

\section{Table 1}

Oligonucleotide primers used for RT-PCR

\begin{tabular}{lll}
\hline GAPDH & Sense & 5'-TCCACCACCCTGTTGCTGTAG-3' \\
Tropoelastin & Anti-sense & 5'-GACCACAGTCCATGCCATCACT-3' \\
& Sense & 5'-GGTGCGGTGGTTCCTCAGCCTGG-3' \\
Fibrillin-1 & Anti-sense & 5'-GGGCCTTGATGGGATACCCCAGTG-3' \\
& Sense & 5'-AATGTGCTTTCAATTGATG-3' \\
Fibulin-1 & Anti-sense & 5'-ATTACCACTTGGTGTATA-3' \\
& Sense & 5'-GGAATCCAAAGAATGCAGGA-3' \\
Fibulin-4 & Anti-sense & 5'-ACTGGTAGCCACCATGAGG-3' \\
& Sense & 5'-ATGCGAGCAGCGCTGCTTCAA-3' \\
Collagen-I & Anti-sense & 5'-AGCCCCCATGGAAGTTGACAC-3' \\
& Sense & 5'-CCCACCAATCACCTGCGTACAGA-3' \\
& Anti-sense & 5'-TTCTTGGTCGGTGGGTGACTCTGA-3'
\end{tabular}

Oligonucleotide primers used for real-time RT-PCR

\begin{tabular}{lll}
\hline GAPDH & Sense & 5'-TCAACAGCGACACCCACTCC-3' \\
Tropoelastin & Anti-sense & 5'-TGAGGTCCACCACCCTGTTG-3' \\
& Sense & 5'-GCTGACGCTGCTGCAGCCTA-3' \\
Fibrillin-1 & Anti-sense & 5'-CAGCAAAAGCTCCACCTACA-3' \\
& Sense & 5'-TGACTGGCCCACACGTGCATAG-3' \\
Fibulin-1 & Anti-sense & 5'-TGACATTGACCCCTTGTTGACAGGA-3' \\
& Sense & 5'-GGAGCAGTGCTGCCACAG-3' \\
Fibulin-4 & Anti-sense & 5'-AGCACCTCTTCACAAATGTG-3' \\
& Sense & 5'-ATGCGAGCAGCGCTGCTTCAA-3' \\
& Anti-sense & 5'-AGCCCCCATGGAAGTTGACAC-3' \\
\hline
\end{tabular}

(Applied Biosystems, Foster City, CA, USA) and carried out in 7000 Sequence Detection System at $50{ }^{\circ} \mathrm{C}$ for $2 \mathrm{~min}$ and then at $95^{\circ} \mathrm{C}$ for $10 \mathrm{~min}$, followed by 40 cycles of amplification $\left(95^{\circ} \mathrm{C}\right.$ for $15 \mathrm{~s}$ and $60^{\circ} \mathrm{C}$ for $1 \mathrm{~min}$ ). The primers used are listed in Table 1 . In each presented case, three separate reactions were performed and the levels of particular ECM component-encoding mRNAs were normalized to the amount of GAPDH mRNA. In both assays, the mean and SD were calculated. Student's $t$-test was used to calculate the $P$-value. The $P$-value of $<0.05$ was considered significant.

\section{Quantitative assay of the newly produced insoluble elastin}

All LDS fibroblasts were grown to confluency in $30 \mathrm{~mm}$ dishes and then cultures were maintained for the next 3 days in fresh media (DMEM with $2 \%$ FBS) in the presence and absence of $10 \mu \mathrm{M}$ dexamethasone. Aliquots of $20 \mu \mathrm{Ci}$ of $\left[{ }^{3} \mathrm{H}\right]$-valine were added to each dish along with fresh media. At the end of the incubation period, the quadruplicate cultures were used for the quantitative assessment of metabolically labeled (radioactive) insoluble elastin as previously described. ${ }^{13}$ Cell layers containing newly deposited matrix were boiled in $0.5 \mathrm{ml}$ of $0.1 \mathrm{~N} \mathrm{NaOH}$ for $45 \mathrm{~min}$ to solubilize all matrix components except elastin. The collected insoluble radioactive elastin was then solubilized by boiling in $200 \mu \mathrm{l}$ of $5.7 \mathrm{~N} \mathrm{HCl}$ for $1 \mathrm{~h}$ and quantified in scintillation counter. Aliquots taken from each culture were also used for DNA determination using the DNeasy Tissue System from Qiagen. Final results reflecting amounts of metabolically labeled insoluble (crosslinked) elastin in individual cultures were normalized per their DNA content and expressed as counts per min per $1 \mu \mathrm{g}$ of DNA.

\section{Immunostaining}

The parallel cultures maintained for 7 days in the presence and absence of $10 \mu \mathrm{M}$ dexamethasone, which was added on day 3 and day 5 , were either fixed in ice-cold $100 \%$ methanol at $-20^{\circ} \mathrm{C}$ (for elastin or fibrillin detection) or in $4 \%$ paraformaldehyde at room temperature (for collagen detection) for $30 \mathrm{~min}$ and blocked with $1 \%$ normal goat serum for $1 \mathrm{~h}$. The cultures were then incubated for $1 \mathrm{~h}$, either with $10 \mu \mathrm{g} / \mathrm{ml}$ polyclonal antibody to tropoelastin or monoclonal 
antibody to fibrillin 1 (both from Elastin Products, Owensville, MO, USA), $5 \mu \mathrm{g} / \mathrm{ml}$ antibody to fibulin 1 (Chemicon, Billerica, MA, USA), $1 \mu \mathrm{g} / \mathrm{ml}$ antibody to fibulin 4 (Santa Cruz Biotechnology, Santa Cruz, CA, USA) or with $10 \mu \mathrm{g} / \mathrm{ml}$ polyclonal antibody to collagen type I (Chemicon). All cultures were then incubated for an additional hour with the appropriate fluorescein-conjugated secondary antibodies (Sigma). Nuclei were counterstained with propidium iodide (Sigma). Secondary antibodies alone were also used as an additional control. All cultures were examined with a Nikon Eclipse E1000 microscope attached to a cooled charge-coupled device camera (Retiga EX; QImaging, Surrey, BC, Canada). All immunodetected ECM components were quantitatively analyzed with the computer-generated video analysis system (Image-Pro Plus software; Media Cybernetics, Silver Spring, MD, USA). In each group, 20 low-power fields $(\times 20)$ from three separate cultures were analyzed, and the areas occupied by the particular immunodetectable components were

a Levels of mRNAs encoding Components of Elastic Fibers in 24 hour-old cultures of Dermal Fibroblasts One step RT-PCR
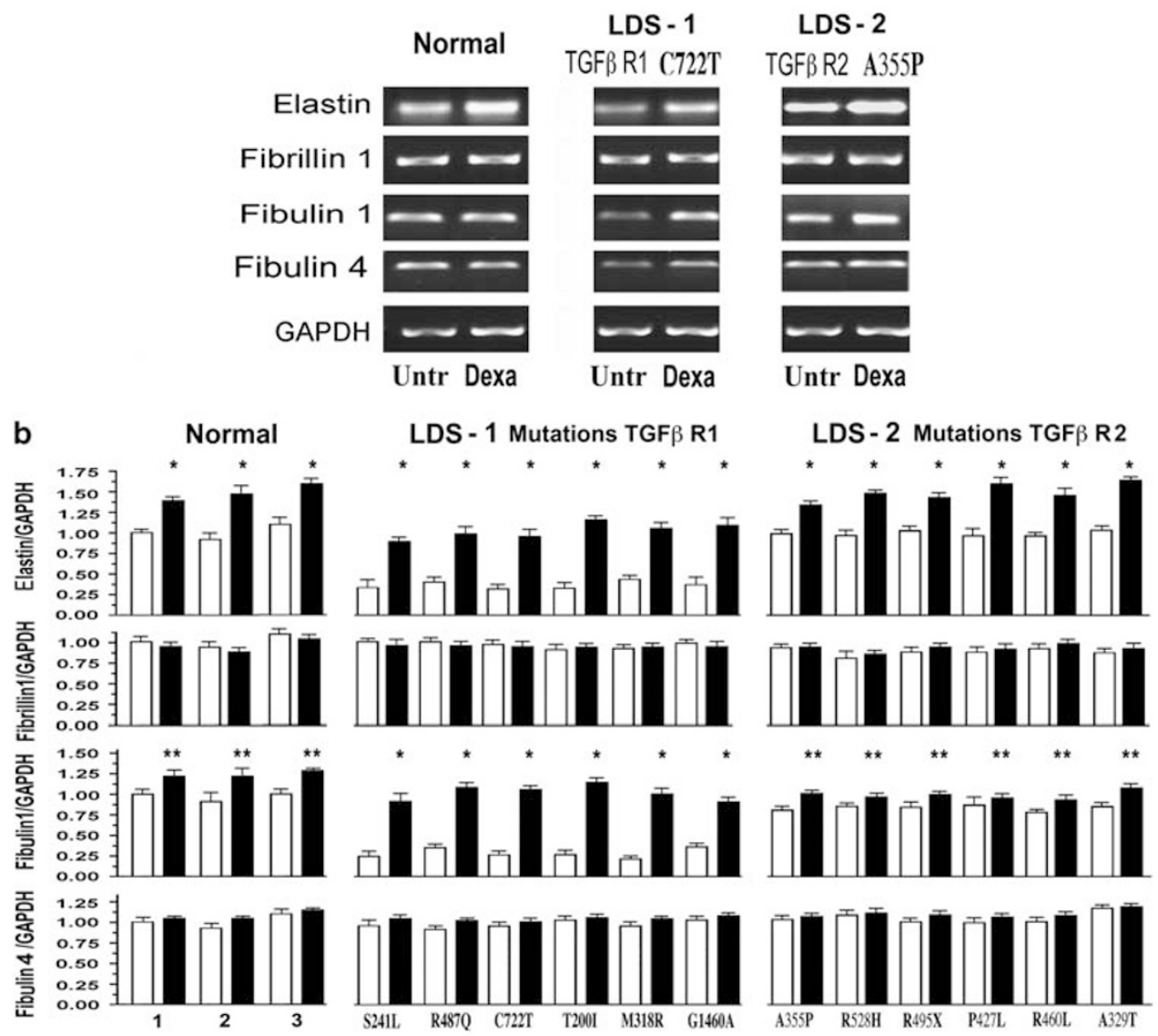

C

Real Time RT-PCR
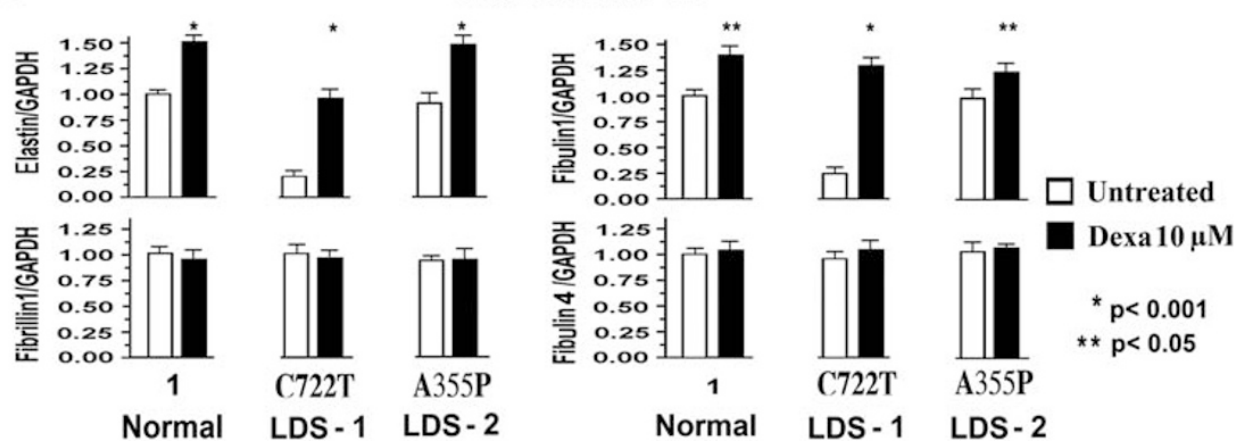

Figure 1 Representative images of RT-PCR analysis (a) and quantification of one-step RT-PCR evaluation of mRNA levels encoding components of elastic fibers in 24-h-old cultures of normal fibroblasts and fibroblasts derived from 12 patients with LDS (b). The $24 \mathrm{~h}$-long treatment with $10 \mu \mathrm{m}$ dexamethasone (Dexa) significantly upregulated the abnormally low levels of mRNAs encoding tropoelastin and fibulin 1 observed in untreated cultures of fibroblasts derived from patients with LDS-1 and further enhanced the basic levels of mRNAs encoding these components of elastic fibers in normal and LDS-2 fibroblasts. Treatment with dexamethasone did not induce any increase in the steady-state levels of mRNAs encoding fibrillin 1 and fibulin 4. (c) Results of the real-time RT-PCR estimation of mRNAs encoding ECM components, performed in representative cultures of normal fibroblasts and fibroblasts derived from patients with LDS 1 and LDS 2, were proportionally similar to results obtained by the one-step RT-PCR. 
Components of Elastic Fibers detected by Immunostaining in 7-day-old cultures of Dermal Fibroblasts

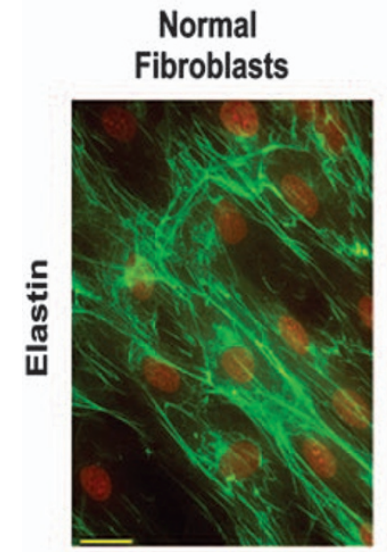

LDS Fibroblasts with TGF $\beta R 1$ mutation

S241L
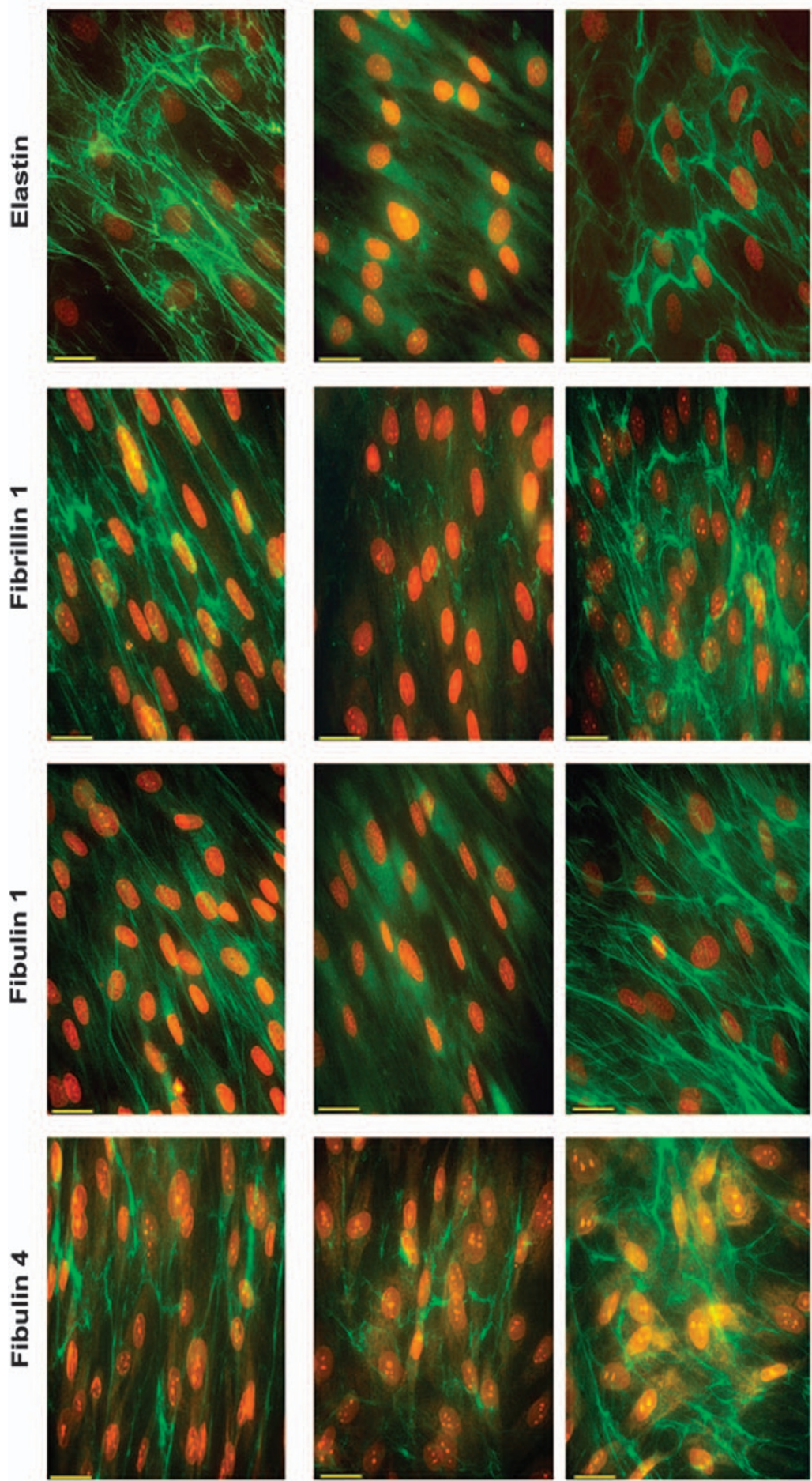

Untreated
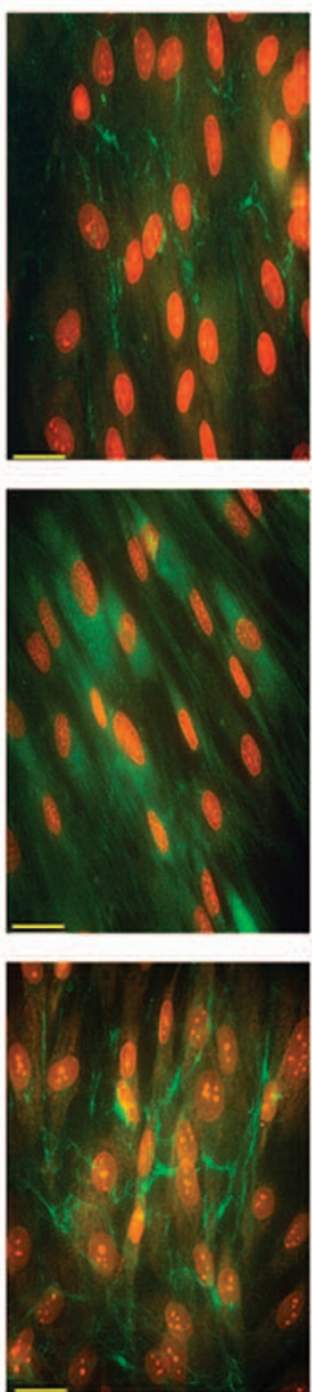

Untreated
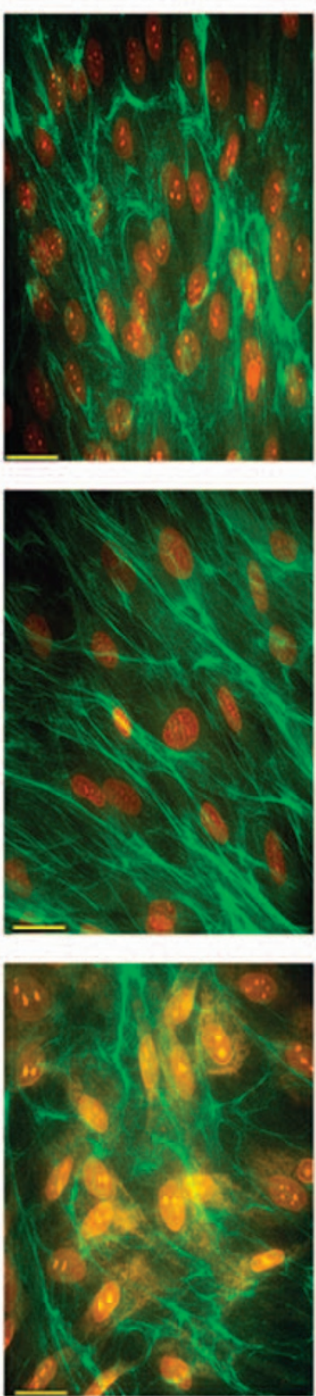

Dexamethasone $10 \mu \mathrm{M}$
LDS Fibroblasts with TGFBR2 mutation P427L
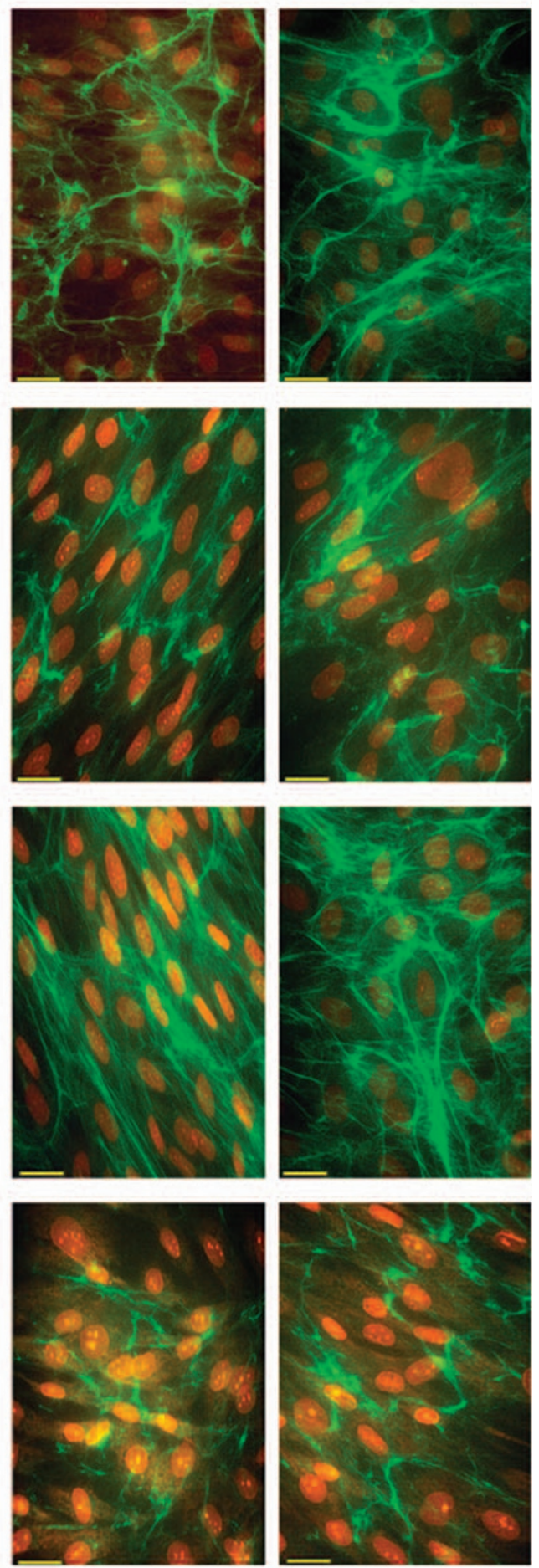

Untreated

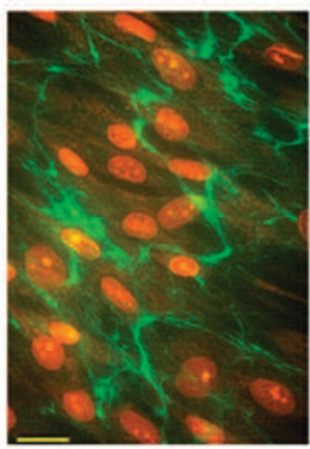

Dexamethasone $10 \mu \mathrm{M}$

Figure 2 Representative micrographs depicting 7-day-old cultures of normal skin fibroblasts and LDS fibroblasts immunostained with antibodies specific to indicated components of elastic fibers (scale bars $=10 \mu \mathrm{m}$ ). The LDS-1 fibroblasts bearing TGF $\beta$-R1 gene mutations deposited only negligible amounts of immunodetectable elastin, fibrillin 1 , fibulin 1 and fibulin 4 when compared with normal fibroblasts and LDS-2 fibroblasts with TGF $\beta$-R1 gene mutations. All dexamethasone-treated cultures of LDS fibroblasts proportionally improved their net deposition of all tested proteins and assured their assembly into thick elastic fibers. 
quantified. In cultures probed with antibody-recognized collagen type I, we separately estimated the green fluorescence present inside and between cells. Then, the percentage of fluorescence detected in both compartments was estimated, relatively to the total fluorescence detected in the given analyzed field.

\section{Electron microscopy}

Four randomly selected 5-day-old cultures of LDS fibroblasts that were maintained in the presence and absence of $10 \mu \mathrm{M}$ dexamethasone were fixed in $2 \%$ glutaraldehyde in $0.1 \mathrm{M}$ cacodylate buffer containing $2.5 \%$ tannic acid, postfixed with $1 \%$ osmium tetroxide in the same buffer, dehydrated in ethanol and embedded in Epon. This preparation gave high contrast of elastin when thin sections were stained with uranyl acetate and lead citrate.

\section{RESULTS}

Dermal fibroblasts derived from LDS patients displayed abnormal pattern of expression of genes encoding elastin and fibulin 1

Initial RT-PCR-based comparison of primary cultures of dermal fibroblasts revealed that fibroblasts derived from six LDS patients with different TGF $\beta$-R1 mutations consistently demonstrated an average $72 \pm 6 \%$ less tropoelastin-encoding mRNA and $87 \pm 6 \%$ less fibulin 1-encoding mRNA than normal fibroblasts (Figure 1). In contrast, fibroblasts from six LDS patients with mutations of the TGF $\beta$-R2 gene showed normal levels of tropoelastin mRNA and only $9 \pm 4 \%$ less fibulin 1-encoding mRNA than normal fibroblasts. All tested LDS fibroblasts produced near-normal levels of fibrillin 1- and fibulin 4-encoding mRNAs.

\section{Dexamethasone stimulated elastin and fibulin 1 gene expression} RT-PCR analysis showed that treatment of fibroblasts with $10 \mu \mathrm{M}$ dexamethasone for $24 \mathrm{~h}$ returned the initially low levels of elastin and fibulin 1 mRNAs to near-normal levels in all LDS fibroblasts with TGF $\beta$-R1 gene mutations. Dexamethasone also enhanced the transcription of mRNAs for elastin and fibulin 1 in normal fibroblasts and in LDS fibroblasts with mutations of TGF $\beta$-R2 (Figure 1). Treatment with dexamethasone did not induce any increase in the steady-state levels of mRNAs encoding fibrillin 1 and fibulin 4. In contrast, all fibroblasts treated with dexamethasone demonstrated a slight decrease in the levels of mRNA encoding collagen 1 (Figure 4a). Importantly, the results of the additional real-time RT-PCR estimation of mRNAs encoding ECM components, performed in the representative cultures of normal fibroblasts and fibroblasts derived from patients with LDS 1 and LDS 2, were proportionally similar to results obtained by the one-step RT-PCR (Figure 1c).

\section{Dexamethasone induced remarkable improvement in the net deposition of normal elastic fibers and enhanced secretion of collagen 1 in cultures of LDS fibroblasts}

Immunohistochemical staining of parallel 7-day-old cultures of skin fibroblasts with anti-elastin, anti-fibrillin 1, anti-fibulin 1 and anti-fibulin 4 antibodies (Figure 2), followed by morphometric quantitative analysis (Figure 3), showed that LDS fibroblasts with TGF $\beta$-R1 gene mutations deposited only negligible amounts of immunodetectable elastin, fibrillin 1 and fibulin 1 that would be organized into elastic fibers compared with normal fibroblasts. Only fibulin 4 was deposited in the fibrillar form, but the detectable amount was also lower than in cultures of normal fibroblasts. Deposition of all immunodetected elastic fiber components was markedly higher in cultures of fibroblasts with TGF $\beta$-R2 mutations than those with TGF $\beta$-R1 mutations and only slightly lower than those detected in parallel cultures of normal fibroblasts. The results of morphometric evaluation also indicated that fibroblasts with TGF $\beta$-R2 gene mutations demonstrated abnormal intracellular retention of immunodetectable collagen 1, coexistent with a significantly lower number of extracellular collagen fibers than could be detected in cultures of normal fibroblasts or cells with mutations in TGF $\beta$-R1 gene (Figure 4b). All dexamethasone-treated LDS fibroblasts improved their net deposition of all tested components of elastic fibers that organize into normal elastic fibers. The improvement in the elastic fiber deposition following treatment with dexamethasone was particularly striking in cultures of fibroblasts with TGF $\beta$-R1 gene mutations. These data correlated well with results of a quantitative assay measuring deposition of $\left[{ }^{3} \mathrm{H}\right]$-valine-labeled insoluble elastin (Figure 3b).

Dexamethasone treatment also improved the net deposition of extracellular collagen fibers in all cultures of LDS fibroblasts. This effect was particularly impressive in cultures of cells with TGF $\beta$-R2 gene mutations, where dexamethasone rectified the intracellular retention of collagen type I and enhanced a concomitant deposition of collagen fibers outside of the cell in the extracellular matrix. EM confirmed the findings obtained using light microscopy immunohistochemistry (Figure 5).

\section{DISCUSSION}

TGF $\beta 1$ and TGF $\beta 2$ are multipotential cytokines involved in the development of numerous body organs and systems, including craniofacial structures and the cardiovascular system. They interact with different receptors coded by the TGF $\beta$-R1 and TGF $\beta$-R2 genes. The ligand TGF $\beta 1$ binds first to a homodimer of the TGF $\beta$-R2 subunits and then recruits a homodimer of the TGF $\beta$-R1 protein, consecutively activating it by multiple phosphorylation of its glycineserine-rich region. ${ }^{14}$ In turn, the activated TGF $\beta$-R1 protein propagates downstream signals causing phosphorylation of the Smad family members, the known regulators of transcription of multiple genes. Despite numerous reports of different mutations in the TGF $\beta$ receptor genes and the variability of clinical manifestations associated with LDS, little is known about the cellular mechanisms responsible for development of particular phenotypic features. The existence of significant phenotypic overlap between LDS and MFS suggests that the causative mechanism may be similar in both conditions. Paradoxically increased TGF $\beta$ signaling has a significant role in MFS and it has been suggested that increased TGF $\beta$ signaling also contributes to other MFS-like disorders, including LDS. Increased TGF $\beta$ signaling is thought to be triggered by the absence of fully assembled fibrillin 1 , a protein that normally sequesters TGF $\beta$, thereby contributing to downregulation of its functional potential. There is solid evidence that the genomic fibrillin 1 deficiency in MFS results in end-organ damage via this route. ${ }^{9,15}$ It has been also documented that TGF $\beta$ signaling can be blocked by losartan, an angiotensin II type I receptor antagonist, resulting in rescue of aortic dilatation in the Marfan transgenic mouse model, leading to a randomized controlled trial of losartan in human MFS patients. ${ }^{16,17}$ It has been also suggested that mutations in TGF $\beta$ receptor-encoding genes (TGF $\beta$-R1 and 2 ) would lead to persistent and increased TGF $\beta$-induced signals in LDS patients. ${ }^{1}$ However, this suggestion does not correlate well with the fact that TGF $\beta$ can normally induce transcription of elastin gene and enhance elastin mRNA stability, as well as induce transcription genes encoding lysyl oxidases (LOXes) that are responsible for crosslinking of both elastin and collagen precursors. ${ }^{18-20}$ It is not clear yet whether the initially impaired fetal elastogenesis and aberrant collagen deposition directly contribute to early aortic dissections in children with LDS. ${ }^{11}$ However, the result of our study provides a novel insight into the pathomechanism of these features. We have demonstrated a significant reduction 


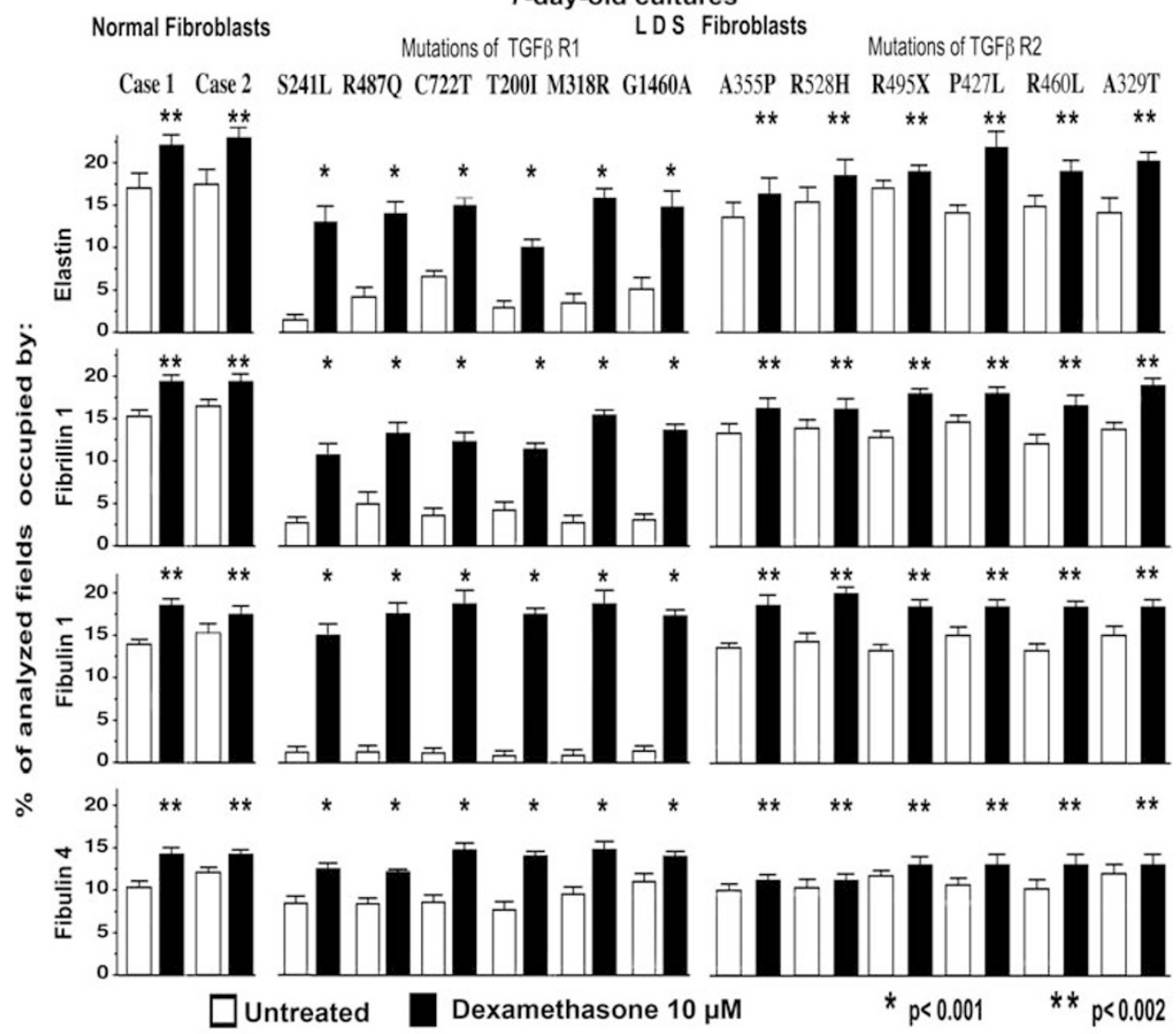

b

Deposition of metabolicaly labeled Insoluble Elastin in 7-day-old cultures L D S Fibroblasts

Mutations of TGF $\beta$ R1

Mutations of TGF $\beta$ R2

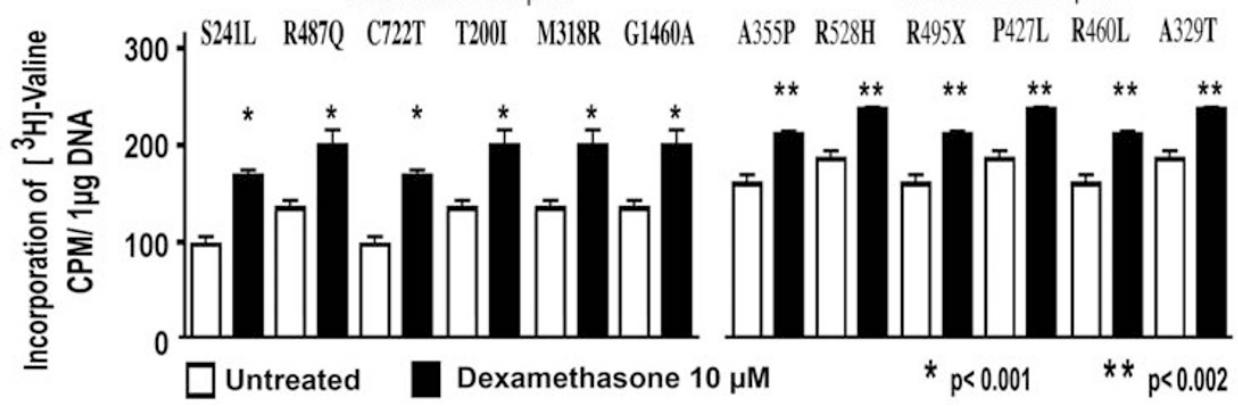

Figure 3 (a) Results of the morphometric quantitative analysis confirmed that levels of net deposition of immunodetected elastin, fibrillin 1, fibulin 1 and fibulin 4 in untreated 7-day-old cultures of LDS-1 fibroblasts with six different mutations of TGF $\beta$-R1 were consistently and significantly lower than those observed in the cultures of normal and LDS-2 fibroblasts. The results also confirm that all dexamethasone-treated cultures proportionally improved their net deposition of all four elastic fiber components. (b) Results of a quantitative assay measuring incorporation of $\left[{ }^{3} \mathrm{H}\right]$-valine into the insoluble elastin demonstrate that cultured LDS fibroblasts carrying mutations of both TGF $\beta$ receptor-encoding genes produced more insoluble elastin in response to dexamethasone treatment.

in the deposition of elastic fibers in cultured LDS fibroblasts with TGF $\beta$ R1 gene mutations and faulty deposition of collagen fibers in the cultures of LDS fibroblasts with TGF $\beta$-R2 gene mutations. Most importantly, for the first time we have established that treatment with dexamethasone leads to a remarkable reversal of these pathological features observed in both types of LDS fibroblasts. Although the detailed analysis of all TGF $\beta$ signaling pathways was beyond the scope of the present study, we may only speculate that the reported abnormal deposition of extracellular matrix components occur in result of either an inadequate or an imbalanced transduction of TGF $\beta$ signaling in analyzed LDS fibroblasts bearing mutated TGF $\beta$ receptors. We have shown consistently lower than normal expression of elastin and fibulin 1 genes in fibroblasts derived from six LDS patients with TGF $\beta$ R1 mutations. Despite the fact that all tested LDS fibroblasts 
a

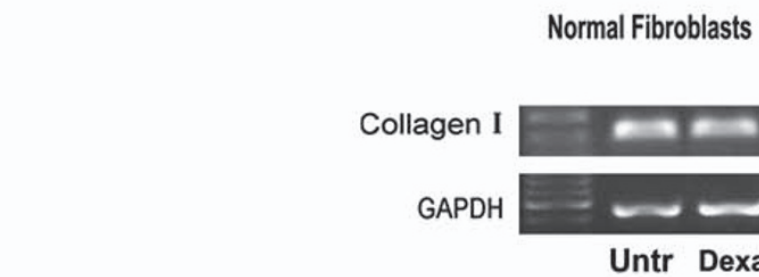

Levels of mRNAs encoding Collagen type I $\alpha 1$ chain

LDS Fibroblasts

TGFBR1 C722T TGF $\beta$ R2 A355P

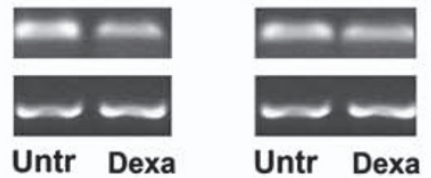

Normal Fibroblasts

Mutations of TGF $\beta$ R1

LD S Fibroblasts

Mutations of TGF $\beta$ R2

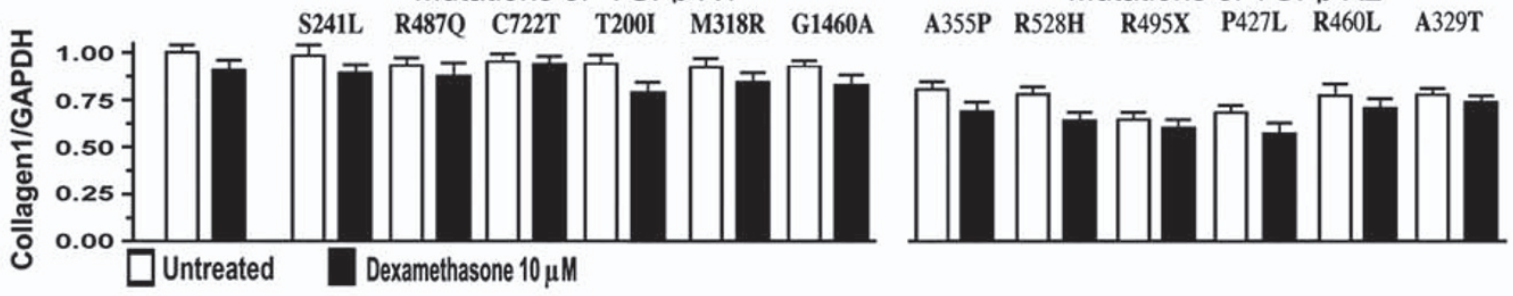

b

Immuno-detection of Collagen I in 7-day-old cultures

Normal Fibroblasts

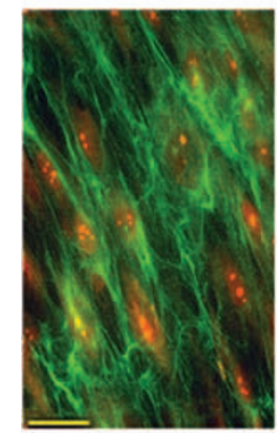

Untreated

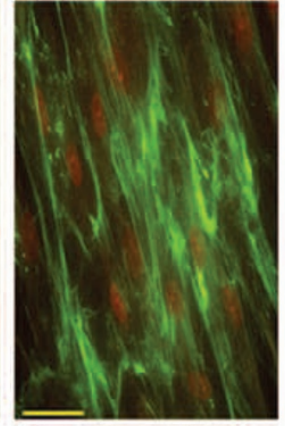

Dexamethasone $10 \mu \mathrm{M}$
LDS Fibroblasts TGF $\beta$ R1 mutation S241L

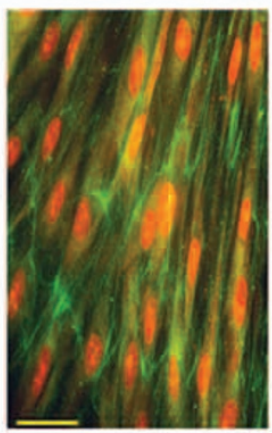

Untreated

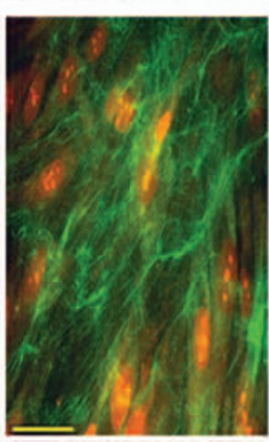

Dexamethasone $10 \mu \mathrm{M}$
TGF $\beta$ R2 mutation R528H

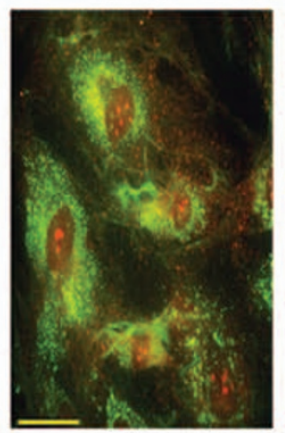

Untreated

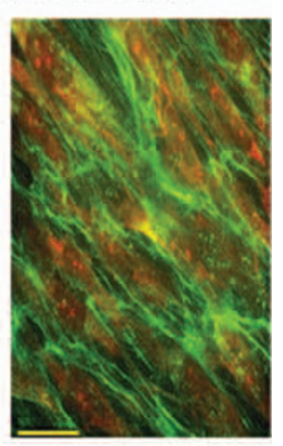

Dexamethasone $10 \mu \mathrm{M}$

Morphometric analysis of immuno-detected Collagen I

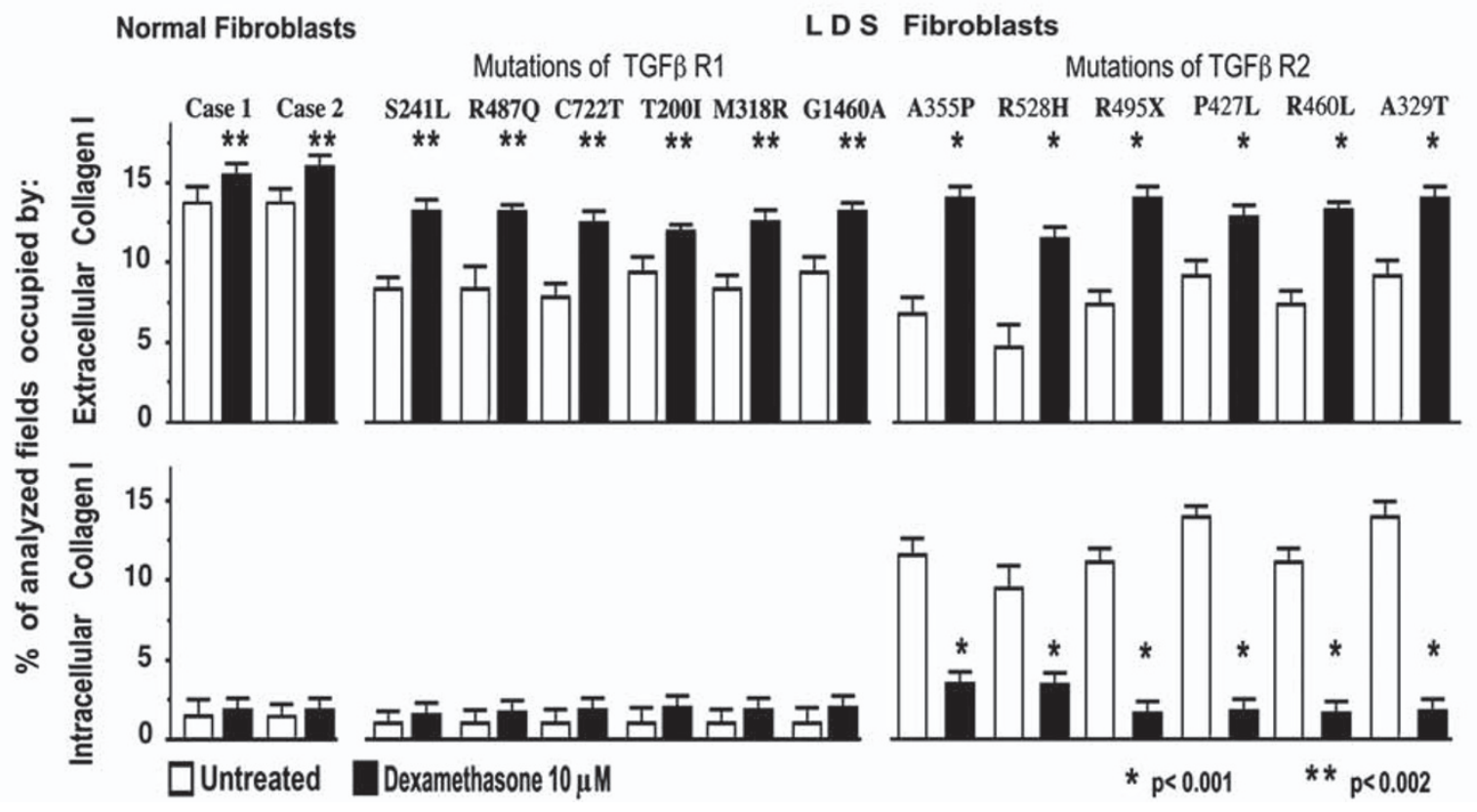



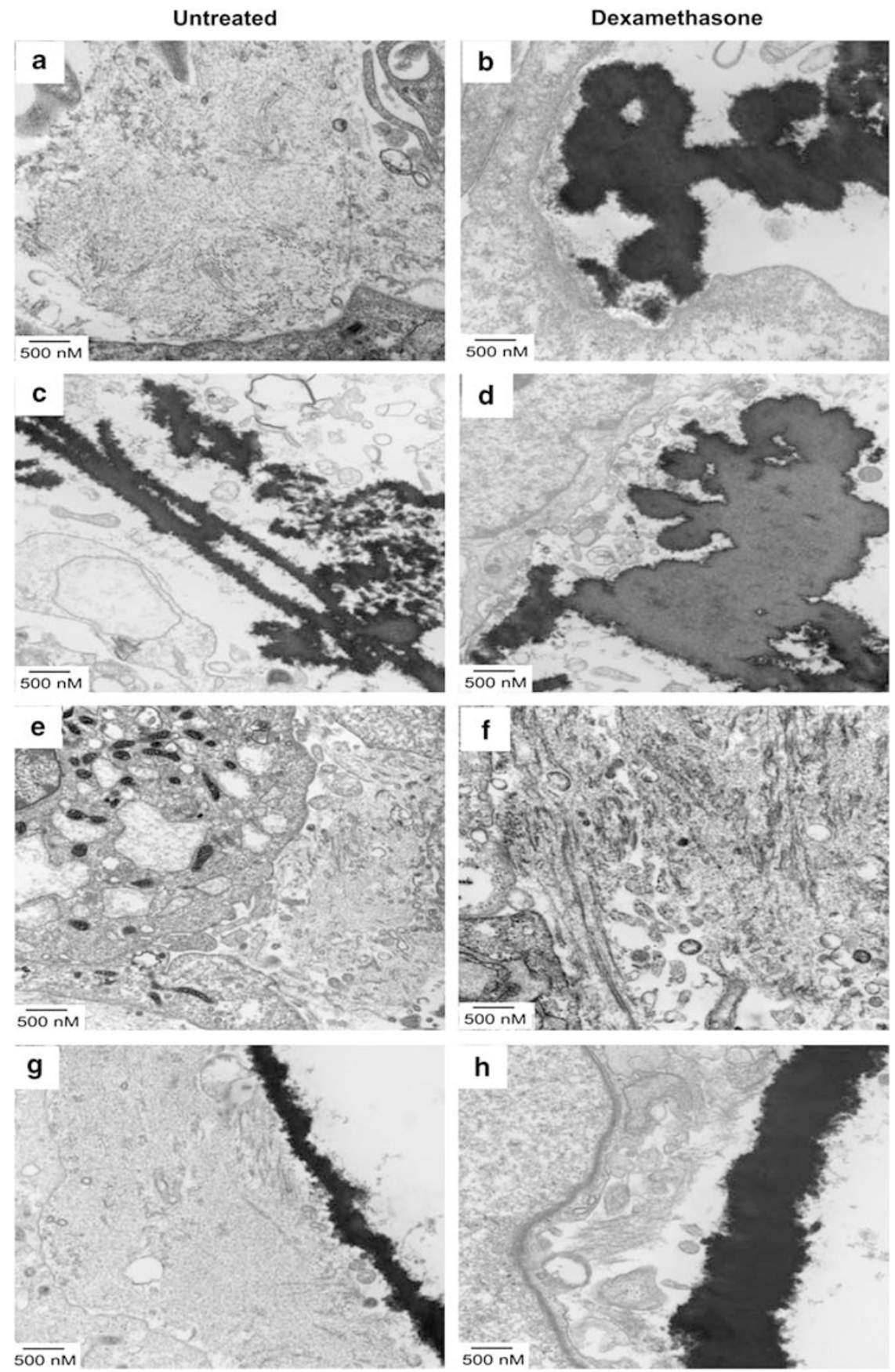

Figure 5 The representative EM micrographs demonstrate that the matrix produced by cultured LDS fibroblasts with TGF $\beta$-R1 gene mutation consists of tightly packed collagen fibers and a striking lack of elastic fibers (a) and that dexamethasone treatment induced restoration of elastic fibers (b). Fibroblasts bearing mutation of TGF $\beta$-R2 gene that produced thin and immature elastic fibers, when cultured in normal medium (c), demonstrated thick and wellorganized elastic fibers, following dexamethasone treatment (d). In contrast to untreated LDS fibroblasts with TGF $\beta$-R2 mutation that show a storage of fibrous collagen material in intracellular vacuoles and only single thin collagen fibers in ECM (e), the cultures treated with dexamethasone demonstrate normal collagen fibers in the extracellular space (f). Cultures of normal fibroblasts treated with dexamethasone (h) also produce more extracellular collagen and thicker elastic fibers than their untreated counterparts $(\mathbf{g})$.

Figure 4 (a) Images of the representative RT-PCR analysis (upper panel) and results of a quantitative RT-PCR evaluation (lower panel) demonstrate that fibroblasts derived from all 12 patients with LDS show almost similar steady-state levels of mRNAs encoding collagen type I $\alpha 1$ chain as parallel cultures of normal fibroblasts, and that 24-h-long treatment with dexamethasone (Dexa) leads to a slight decrease in the levels of mRNA encoding collagen type 1. (b) Representative micrographs depicting cultures immunostained with anti-collagen type I antibody (upper panel) (scale bars $=20 \mu \mathrm{m}$ ) and results of morphometric evaluation of all immunostained cultures (lower panel) indicate that fibroblasts with TGF $\beta$-R2 gene mutations show an abnormal intracellular retention of immunodetectable collagen type I, coexisting with a significantly lower number of extracellular collagen fibers that could be detected in cultures of normal fibroblasts or fibroblasts bearing TGF $\beta$-R1 gene mutations. Dexamethasone treatment rectified the abnormal intracellular retention of collagen I in fibroblasts with TGF $\beta$-R2 mutations and improved the net deposition of extracellular collagen fibers in all cultures of tested LDS fibroblasts. 
with TGF $\beta$ R1 mutations revealed normal levels of the steady-state expression of fibrillin 1 and fibulin 4 genes, they ultimately demonstrated a marked decrease in deposition of immunodetected microfibrils. This coincided with the conspicuously low extracellular deposition of insoluble (crosslinked) elastin and lack of normal assembly of immunodetected elastic fibers. The possible involvement of aberrant TGF $\beta$ signaling, potentially interfering with the net production of microfibrils, is not entirely clear at this point. However, our data strongly indicate that the presence of mutations in TGF $\beta$-R1, but not mutations in TGF $\beta$-R2, effectively interferes with the propagation of the elastogenic signals normally induced by TGF $\beta$.

Up to eight different transcription start sites have been identified in the elastin gene, indicating that the transcription of this gene is controlled by complex mechanisms. Numerous factors and hormones such as TNFa1, insulin-like growth factor I, interleukin-1 $\beta$ and interleukin 10 have all been shown to induce signals that eventually affect tropoelastin synthesis at the promoter region, after first interacting with their respective cell surface receptors. ${ }^{21}$ Glucocorticoids, however, can effectively by-pass cell membrane receptors and interact directly with elastin gene regulatory elements to produce upregulation of the elastin gene transcription. ${ }^{12}$ It has been specifically demonstrated that glucocorticoid-induced signaling can use alternative pathways and exert its effect without involvement of TGF- $\beta$-dependent signals. ${ }^{22}$ With this in mind, we suggest that dexamethasone, through a direct interaction with the promoter region of the elastin gene, might serve as an alternative elastogenic stimulator for LDS fibroblasts bearing mutated TGF $\beta$-R1 that are apparently unable to properly propagate the TGF $\beta$-induced elastogenic signals. ${ }^{23}$ At the same time, we documented that treatment with dexamethasone also induced a consistent increase in deposition of major components of microfibrillar scaffold of elastic fibers, fibulin 1 and fibrillin 1 . Although the recovery of fibulin 1 microfibrils (Figure 2) can be linked to the observed (Figure 1) and previously documented ${ }^{24}$ primary stimulation of fibulin 1 gene expression by dexamethasone, the subsequent remarkable increase in the deposition of fibrillin 1 microfibrils has not been preceded by any increase in fibrillin 1 mRNA level. As the basic expression of fibrillin 1 was not substantially harmed in LDS fibroblasts bearing mutated TGF $\beta$-R1, we may additionally speculate that dexamethasone would also alleviate an aberrant signaling contributing to under-expression or inactivation of furin. This protein convertase is normally responsible for the posttranslational processing of pro-fibrillin 1 molecules, the prerequisite for their homo-polymerization and assembly into the microfibrillar scaffold of elastic fibers. ${ }^{25,26}$

Interestingly, the apparent aberration in TGF $\beta$ signaling in LDS fibroblasts did not affect the expression of collagen 1. However, we found that all six fibroblasts with TGF $\beta$-R2 mutations demonstrated lower than normal deposition of extracellular collagen fibers and a peculiar intracellular accumulation of fibrous material detectable with antibody to collagen type I. This observation suggests that abnormal TGF $\beta$-generated signaling may result in either inhibition of the proper intracellular processing and secretion of collagen I precursors, or with the enhancement of phagocytosis of the already secreted tropocollagen molecules, which could not be properly crosslinked in the extracellular space. The latter possibility seems to be more probable as the LDS fibroblasts demonstrated lower than normal expression of LOX and a similar intracellular accumulation of collagen 3 has been described as a cardinal feature in EDS vascular type, characterized by LOX deficiency. ${ }^{27}$

Currently, we are unable to exclude the involvement of other parallel mechanisms that could be triggered by dexamethasone, and further studies are needed for the detailed elucidation of the phenomenon observed in our in vitro studies. It would be particularly rational to learn, for example, whether dexamethasone would also upregulate expression of the non-mutated alleles encoding normal TGF $\beta$ receptors in the heterozygotic LDS patients.

In summary, we show that alterations of normal elastogenesis and collagen I deposition heavily contribute to the LDS phenotypes. Most importantly, observation of the remarkable improvement in deposition of both fibrillar ECM components following treatment with dexamethasone raises the intriguing question of whether prenatal treatment with this drug may lead to amelioration of the LDS phenotypes. Prenatal dexamethasone treatment may have practical utility because LDS can be diagnosed prenatally ${ }^{28}$ and fetal LDS patients may be identified via their affected parent. The possibility of prenatal treatment is particularly important in light of the fact that organogenesis-relevant elastogenesis occurs predominantly in the last trimester of human fetal life and in the early weeks of postnatal life. $^{21,29}$ This provides a potential window of opportunity for prenatal treatment with dexamethasone in cases at risk or known to be affected with LDS. The observation that dexamethasone ameliorated intracellular storage of collagen type I and normalized deposition of collagen fibers in fibroblasts with mutations in the TGF $\beta$-R2 gene additionally supports our suggestion that early prenatal treatment with dexamethasone may alleviate connective tissue problems observed in LDS patients.

Dexamethasone has been already safely and effectively used prenatally to suppress adrenal gland activity in cases with congenital adrenal hyperplasia in female fetuses. ${ }^{30}$ The safety of pulse-dose corticosteroids has been established in pregnancy through their widespread use over the past 15 years in the prevention of prematurityrelated respiratory distress syndrome and associated morbidities. ${ }^{31,32}$ Furthermore, short-term treatment of LDS patients before vascular surgery may improve the strength of aortic wall, diminish the risk of aneurysm rupture and potentially increase the successful outcome of the surgical procedure.

\section{CONFLICT OF INTEREST}

The authors declare no conflict of interest.

\section{ACKNOWLEDGEMENTS}

This work was supported by the Canadian Institute of Health Research through grant PG 13920 and by the Heart and Stroke Foundation of Ontario through grant NA 5435 to AH. We are grateful to Dr H Dietz from Johns Hopkins Medical Center, USA, for sharing fibroblasts derived from his LDS patients.

1 Loeys BL, Chen J, Neptune ER et al: A syndrome of altered cardiovascular, craniofacial, neurocognitive and skeletal development caused by mutations in TGFBR1 or TGFBR2. Nat Genet 2005; 37: 275-281.

2 Hanks SK, Hunter T: Protein kinases 6. The eukaryotic protein kinase superfamily: kinase (catalytic) domain structure and classification. FASEB J 1995; 9: 576-596.

3 Chang YC, Yang SF, Lai CC, Liu JY, Hsieh YS: Regulation of matrix metalloproteinase production by cytokines, pharmacological agents and periodontal pathogens in human periodontal ligament fibroblast cultures. J Periodontal Res 2002; 37: 196-203.

4 Drera B, Tadini G, Barlati S, Colombi M: Identification of a novel TGF $\beta R 1$ mutation in a Loeys-Dietz syndrome type II patient with vascular Ehlers-Danlos syndrome phenotype. Clin Genet 2008; 73: 290-293.

$5 \mathrm{Ki}$ CS, Jin DK, Chang SH et al: Identification of a novel TGF $\beta$ R2 gene mutation in a Korean patient with Loeys-Dietz aortic aneurysm syndrome; no mutation in TGFßR2 gene in 30 patients with classic Marfan's syndrome. Clin Genet 2005; 68: 561-563.

6 Loeys BL, Schwarze U, Holm T et al: Aneurysm syndromes caused by mutations in the TGF-beta receptor. N Engl J Med 2006; 355: 788-798. 
7 Singh KK, Rommel K, Mishra A et al: TGFBR1 and TGFBR2 mutations in patients with features of Marfan syndrome and Loeys-Dietz syndrome. Hum Mutat 2006; 27: 770-777.

8 Isogai Z, Ono RN, Ushiro S et al: Latent transforming growth factor beta-binding protein 1 interacts with fibrillin and is a microfibril-associated protein. J Biol Chem 2003; 278: 2750-2757.

9 Neptune ER, Frischmeyer PA, Arking DE et al: Dysregulation of TGF-beta activation contributes to pathogenesis in Marfan syndrome. Nat Genet 2003; 33: 407-411.

10 Ng CM, Cheng A, Myers LA et al: TGF-beta-dependent pathogenesis of mitral valve prolapse in a mouse model of Marfan syndrome. J Clin Invest 2004; 114: 1586-1592.

11 Maleszewski JJ, Miller DV, Lu J, Dietz HC, Halushka MK: Histopathologic findings in ascending aortas from individuals with Loeys-Dietz syndrome (LDS). Am J Surg Pathol 2009; 33: 194-201.

12 Ledo I, Wu M, Katchman S et al: Glucocorticosteroids up-regulate human elastin gene promoter activity in transgenic mice. J Invest Dermatol 1994; 103: 632-636.

13 Urbán Z, Riazi S, Seidl TL et al: Connection between elastin haploinsufficiency and increased cell proliferation in patients with supravalvular aortic stenosis and Williams-Beuren syndrome. Am J Hum Genet 2002; 71: 30-44.

14 Wrana JL, Attisano L, Wieser R, Ventura F, Massague J: Mechanism of activation of the TGF-beta receptor. Nature 1994; 370: 341-347.

$15 \mathrm{Ng} \mathrm{CM}$, Cheng A, Myers LA et al: TGF-beta-dependent pathogenesis of mitral valve prolapse in a mouse model of Marfan syndrome. J Clin Invest 2004; 114: 1586-1592.

16 Brooke BS, Habashi JP, Judge DP, Patel N, Loeys B, Dietz HC: Angiotensin II blockade and aortic-root dilation in Marfan's syndrome. N Engl J Med 2008; 358: 2787-2795.

17 Habashi JP, Judge DP, Holm TM et al: Losartan, AT1 antagonist, prevents aortic aneurysm in a mouse model of Marfan syndrome. Science 2006; 312: 117-121.

18 Boak AM, Roy R, Berk J et al: Regulation of lysyl oxidase expression in lung fibroblasts by transforming growth factor-beta 1 and prostaglandin E2. Am J Respir Cell Mol Biol 1994; 11: 751-755.

19 Koslowski R, Seidel D, Kuhlisch E, Knoch KP. Evidence for the involvement of TGFbeta and PDGF in the regulation of prolyl 4-hydroxylase and lysyloxidase in cultured rat lung fibroblasts. Exp Toxicol Pathol 2003; 55: 257-264.
20 Bose KK, Chakraborty J, Khuder S, Smith-Mensah WH, Robinson J: Lysyl oxidase activity in the cells of flexor retinaculum of individuals with carpal tunnel syndrome. J Occup Environ Med 2000; 42: 582-587.

21 Vrhovsky B, Weiss AS: Biochemistry of tropoelastin. Eur J Biochem 1998; 258: 1-18.

22 Shi W, Heisterkamp N, Groffen J, Zhao J, Warburton D, Kaartinen V: TGF-beta 3-null mutation does not abrogate fetal lung maturation in vivo by glucocorticoids. Am J Physiol 1999; 277: L1205-L1213.

23 Kuang P-P, Zhang X-H, Rich CB, Foster JA, Subramanian M, Goldstein RH: Activation of elastin transcription by transforming growth factor- $\beta$ in human lung fibroblasts. Am J Physiol Lung Cell Mol Physiol 2007; 292: L944-L952.

24 Ishibashi T, Takagi $\mathrm{Y}$, Mori $\mathrm{K}$ et al: cDNA microarray analysis of gene expression changes induced by dexamethasone in cultured human trabecular meshwork cells. Invest Ophthalmol Vis Sci 2002; 43: 3691-3697.

25 Ashworth JL, Kelly V, Rock MJ, Shuttleworth CA, Kielty CM: Regulation of fibrillin carboxy-terminal furin processing by $\mathrm{N}$-glycosylation, and association of amino- and carboxy-terminal sequences. J Cell Sci 1999; 112: 4163-4171.

26 Raghunath M, Putnam EA, Ritty T et al: Carboxy-terminal conversion of profibrillin to fibrillin at a basic site by PACE/furin-like activity required for incorporation in the matrix. J Cell Sci 1999; 112: 1093-1100.

27 Holbrook KA, Byers $\mathrm{PH}$ : Ultrastructural characteristics of the skin in a form of the Ehlers-Danlos syndrome type IV. Storage in the rough endoplasmic reticulum. Lab Invest 1981; 44: 342-350.

28 Viassolo V, Lituania M, Marasini M et al: Fetal aortic root dilation: a prenatal feature of the Loeys-Dietz syndrome. Prenat Diagn 2006; 26: 1081-1083.

29 Kielty CM: Elastic fibres in health and disease. Expert Rev Mol Med 2006; 8: 1-23.

30 Nimkarn S, New MI: Prenatal diagnosis and treatment of congenital adrenal hyperplasia owing to 21-hydroxylase deficiency. Nat Clin Pract Endocrinol Metab 2007; 3: 405-413.

31 Kovar J, Willet KE, Hislop A, Sly PD: Impact of postnatal glucocorticoids on early lung development. J Appl Physiol 2005; 98: 881-888.

32 Crowther CA, Doyle LW, Haslam RR, Hiller JE, Harding JE, Robinson JS: Outcomes at 2 years of age after repeat doses of antenatal corticosteroids. N Engl J Med 2007; 357: 1179-1189. 